\title{
Jovens Insatisfeitos com a Imagem Corporal: Estresse, Autoestima e Problemas Alimentares
}

\author{
Andressa Melina Becker da Silva ${ }^{1,2}$ \\ Wagner de Lara Machado 3 \\ Anita Colletes Bellodi ${ }^{2}$ \\ Kainara Silva da Cunha ${ }^{2}$ \\ Sônia Regina Fiorim Enumo² \\ ${ }^{1}$ Universidade de Sorocaba (UNISO), Sorocaba, SP \\ ${ }^{2}$ Pontifícia Universidade Católica de Campinas (PUC-Campinas), Campinas, SP \\ ${ }^{3}$ Pontifícia Universidade Católica do Rio Grande do Sul (PUCRS), Porto Alegre, RS
}

\begin{abstract}
Resumo
Jovens correm riscos para transtornos alimentares. Este estudo verificou as relações entre imagem corporal (IC), estresse percebido, autoestima, comportamento alimentar e Índice de Massa Corporal (IMC) em 238 adolescentes e jovens (14-20 anos; 62,2\% meninas), respondendo ao Eating Atittudes Test, Escala de Estresse Percebido, Silhouette Matching Task e Inventário de Autoestima, medindo-se peso e altura (IMC). Meninas apresentaram riscos para distúrbios alimentares, maior preocupação com IC, mais estresse e menor autoestima; meninos desejam ser mais musculosos. Pela análise de redes, comportamento alimentar e IMC não se associaram, divergindo de outros estudos. Houve relações positivas entre IC e IMC e, entre fator dieta e fator bulimia; foram negativas as relações entre autoestima e estresse, e entre IC e sexo. Foram identificadas relações que podem subsidiar intervenções preventivas e centradas em variáveis, como a preocupação em emagrecer, estresse e autoestima em meninas, e o desejo de aumentar a massa corporal nos meninos.

Palavras-chave: comportamento alimentar, imagem corporal, autoestima, estresse, jovens
\end{abstract}

\begin{abstract}
Young people are at risk for eating disorders. This study verified the relationships between body image (BI), perceived stress, self-esteem, eating behavior, and Body Mass Index (BMI) in 238 adolescents and young adults (14-20 years; 62.2\% girls), using the Eating Attitudes Test, Perceived Stress Scale, Silhouette Matching Task, and Self-Esteem Inventory, measuring weight and height (BMI). Girls were at risk for eating disorders, greater concern about BI, more stress and lower self-esteem; boys showed desire to be more muscular. Results indicated that eating behavior and BMI were not associated, diverging from other studies; there were positive relationships between BI and BMI, and between diet factor and bulimia factor; and there were negative relationships between self-esteem and stress, and between BI and sex. We identified relations that may support preventive interventions, centered on variables such as anxiety about weight loss, stress and self-esteem in girls, and the desire to increase body mass in boys.

Keywords: Eating behavior; body image; self-esteem; stress; young people
\end{abstract}

Young People Dissatisfied with Body Image: Stress, Self-Esteem and Eating Problems

\section{Resumen}

\section{Jóvenes Insatisfechos con la Imagen Corporal: Estrés, Autoestima y Problemas Alimentarios}

Los jóvenes corren riesgos con los trastornos alimentarios. Este estudio verificó las relaciones entre imagen corporal (IC), estrés percibido, autoestima, comportamiento alimentario e Índice de Masa Corporal (IMC) en 238 adolescentes y jóvenes (14-20 años; 62,2\% niñas), respondiendo el Eating Atittudes Test, Escala de Estrés Percibido, Silhouette Matching Task y el Inventario de Autoestima, midiendo peso y altura (IMC). Las niñas presentaron riesgo para disturbios alimentarios, mayor preocupación con IC, más estrés y menor autoestima; los niños desean ser más musculosos. Por el análisis de redes, comportamiento alimentario e IMC no se asociaron, discrepando de otros estudios; hubo relaciones positivas entre IC e IMC, y entre factor dieta y factor bulimia; fueron negativas las relaciones entre autoestima y estrés, y entre IC y sexo. Se identificaron relaciones que pueden subsidiar intervenciones preventivas y centradas en variables, como preocupación en adelgazar, estrés y autoestima en niñas, y deseo de aumentar masa corporal en los niños.

Palabras-clave: Comportamiento Alimentario; Imagen corporal; Autoestima; Estrés; Jóvenes

\section{Introdução}

A busca de adolescentes e jovens pela imagem corporal ideal pode levá-los a atingir valores inadequados de Índice de Massa Corporal (IMC) e apresentar transtornos alimentares. Particularmente os adolescentes, por estarem em processo de formação da identidade, ficam mais susceptíveis às influências de modelos físicos idealizados, veiculados pela mídia e pares. Engajam-se, assim, em comportamentos de risco à saúde, visando a atingir a configuração corporal tida como ideal (Stice \& Shaw, 2002). 
Essa situação é agravada pela exposição dos adolescentes nas mídias sociais, como Facebook, Instagram, dentre outros, em que imagens e trocas de informações entre usuários funcionam como motivadores para a busca do corpo ideal (De Vries, Peter, De Graaf, \& Nikken, 2016; Tiggemann \& Slater, 2015). Há o desejo das mulheres por cirurgias plásticas e procedimentos estéticos, e dos homens, por moldar seu corpo via atividade física intensa (Biolcati, Ghigi, Mameli, \& Passini, 2016). Gondoli, Corning, Salafia, Bucchianeri e Fitzsimmons (2012) acrescentam que o desejo de emagrecer das garotas teria relação com a pressão social por atingir um corpo magro.

Dentre os fatores relacionados ao desenvolvimento de transtornos alimentares e IMC inadequado estão algumas variáveis psicológicas, como o déficit na elaboração da imagem corporal, a baixa autoestima e o maior nível de estresse (Gonçalves, Moreira, \& Fiates, 2013; Gondoli et al., 2012; Miranda, Conti, Bastos, \& Ferreira, 2011). Com um caráter preventivo, esses conceitos são analisados, neste estudo, em uma amostra não clínica de adolescentes, procurando identificar possíveis relações que podem predispor os adolescentes a quadros de transtornos alimentares, apresentados a seguir.

Inicialmente, para estudar essas relações, é básico definir se a pessoa está abaixo do peso, na normalidade, com sobrepeso ou obesidade, usando-se como parâmetro o IMC (Claudat, White, \& Warren, 2016; Grilo, 2014; Murray, Rieger, \& Byrne, 2013). Este é calculado pela medida do peso dividido pela altura ao quadrado (National Institutes of Health, 2000). As alterações no IMC, por sua vez, têm sido associadas aos transtornos alimentares (Dumith \& Farias Júnior, 2010; Fortes, Almeida, \& Ferreira, 2012; Leite et al., 2008), os quais incluem quadros de anorexia e bulimia nervosa, segundo a American Psychiatric Association (APA, 2014).

A anorexia nervosa é caracterizada pela restrição da ingesta calórica, peso corporal baixo em relação à idade, gênero e desenvolvimento, apresentação de medo intenso de ganhar peso ou de engordar, ou o comportamento persistente que interfere no ganho de peso, presença de perturbação na forma de perceber o corpo e ausência persistente de reconhecimento da gravidade do baixo peso (APA, 2014). No outro quadro clínico, na bulimia nervosa, ocorrem episódios recorrentes de compulsão alimentar e comportamentos compensatórios inapropriados recorrentes a fim de impedir o ganho de peso, no mínimo uma vez por semana, durante três meses (APA, 2014). A compulsão alimentar pode ser influenciada por altos níveis de estresse (Gonçalves et al., 2013), que levam a um aumento da concentração de cortisol, o qual estimula a ingestão de alimentos (Gluck, Geliebter, \& Satov, 2001). Considerando-se que a compulsão alimentar é um dos sintomas de bulimia, podendo levar a um aumento do IMC, percebe-se a importância de se avaliar os níveis de estresse de adolescentes e jovens. Assim como a prevenção desses quadros, é necessário observar alguns comportamentos de risco que podem predispor o adolescente a tais patologias, o que tem sido feito pelo Eating Atittudes Test [EAT-26] (Garner, Olmsted, Boher, \& Garfinkel, 1982; adaptado para o português do Brasil por Nunes et al., 1994).

Há, porém, um terceiro quadro, ainda não catalogado como doença no Manual de Diagnóstico de Transtornos Mentais DSM-5 (APA, 2014) a vigorexia. Este quadro envolve uma preocupação de não ser suficientemente forte e musculoso, o que acarreta sérias perturbações no desempenho de atividades sociais ou ocupacionais, pois leva os indivíduos a dedicarem horas do dia a práticas esportivas e à ingestão de anabolizantes (Grilo, 2014; Santos et al., 2012). A autoavaliação quanto ao corpo pode ser influenciada indevidamente pela forma e peso corporais. Mas, essa alteração não ocorre exclusivamente durante episódios de anorexia nervosa, podendo ocorrer também em transtornos dismórficos corporais, como a vigorexia, em homens ou mulheres (APA, 2014).

Estudos apontam diferenças entre o sexo masculino e o feminino quando se trata de risco para transtorno alimentar, com maior risco para o sexo feminino (Alvarenga, Scagliusi, \& Philipp, 2011; Fortes et al., 2015; Gonçalves et al., 2013; Lima et al., 2012; Scherer, Martins, Pelegini, Matheus, \& Petroski, 2010). Além disso, foi identificado, na literatura, que o sexo feminino é o que mais se preocupa com a imagem corporal e possui um comportamento alimentar inadequado (Cordás, Oliveira, Figueiredo, \& Didone, 2007; Costarelli \& Patsai, 2012; Fortes, Amaral, Almeida, \& Ferreira, 2013; Gustafsson, Edllund, Davén, Kjelin, \& Noring, 2010; Lima, Rosa, \& Rosa, 2012; Martini, Assumpção, Barros, Canesqui, \& Barros Filho, 2016; Rawal, Williams, \& Park, 2011). A insatisfação corporal pode ser compreendida como um incômodo que o indivíduo vivencia em relação aos aspectos de sua aparência física (Miranda, Conti, Bastos, \& Ferreira, 2011). Nesse sentido, salienta-se a importância de pesquisas com ambos os gêneros para ter maior compreensão da temática, assim como a inclusão de outras variáveis intervenientes desse processo, visando à integração dessas variáveis. 
Diante do exposto, é relevante estudar como se dão as relações entre algumas variáveis que a literatura aponta como relacionadas ao IMC e aos transtornos alimentares. Pressupõe-se que as diferenças de gênero ocorrem via variáveis intervenientes distintas, cuja rede de relações pode ser evidenciada por meio da análise de rede (network analysis) (Borsboom \& Cramer, 2013), em que todas as variáveis podem ser consideradas simultaneamente. Assim, este estudo pretendeu verificar as relações diretas e apontar direções de fluxos de informação, por meio de análise que indique sistemas de informações entre a imagem corporal, os comportamentos alimentares (que apontam risco para transtornos alimentares), o estresse percebido, a autoestima e o IMC em jovens, com variações para o sexo dos participantes, utilizando-se a análise de rede.

\section{Método}

\section{Participantes}

Participaram do estudo 238 adolescentes e jovens $(62,2 \%$ do sexo feminino), com idade entre 14 e 20 anos $(M=15,44 ; \pm 0,81)$. Todos eram alunos de escolas particulares da cidade de Curitiba, Paraná. Foram incluídos alunos que participavam regularmente das aulas de Educação Física (na qual a avaliação foi realizada), que preencheram corretamente os instrumentos de avaliação e cujos responsáveis assinaram o Termo de Consentimento Livre e Esclarecido. Todos os alunos possíveis participaram do estudo, não sendo excluídos nenhum por questões de algum diagnóstico específico.

\section{Instrumentos}

Para o cálculo do índice de massa corporal (IMC $=$ peso $/$ altura $^{2}$ ), utilizou-se uma balança devidamente calibrada para mensuração do peso e fita métrica para medir a altura. Levaram-se em consideração os valores internacionalmente aceitos, segundo a OMS "World Health Organization" (WHO, 1997): abaixo do peso (IMC $<20 \mathrm{Kg} / \mathrm{m}^{2}$ ), peso normal (IMC entre 20 a 25 $\mathrm{Kg} / \mathrm{m}^{2}$ ), sobrepeso (IMC entre 25 e $30 \mathrm{Kg} / \mathrm{m}^{2}$ ), obesidade (IMC entre 30 e $40 \mathrm{Kg} / \mathrm{m}^{2}$ ) e obesidade mórbida (IMC acima de $40 \mathrm{Kg} / \mathrm{m}^{2}$ ). O IMC tem sido recomendado pela Organização Mundial da Saúde para avaliar estado nutricional de crianças e adolescentes, sendo usado para estudos epidemiológicos (Dumith \& Farias Júnior, 2010; Fortes, Almeida, \& Ferreira, 2012; Leite et al., 2008). Além disso, utilizaram-se os instrumentos descritos a seguir:
1) Eating Atittudes Test [EAT-26] (Garner, Olmsted, Boher, \& Garfinkel, 1982; adaptado para o português do Brasil por Nunes et al., 1994). É composto por 26 itens respondidos em uma escala de seis pontos (sempre, muito frequentemente, frequentemente, algumas vezes, raramente e nunca), como, por exemplo: Fico apavorada com a ideia de estar engordando; Vômito depois de comer; Gosto de sentir meu estômago vazio. O EAT-26 avalia comportamentos alimentares e classifica-os em Padrão Alimentar Anormal, expresso pelo EAT-26 Positivo (escores $\geq 20$ pontos) e em Padrão Alimentar Normal, expresso pelo EAT26 Negativo $(<20$ pontos).

Além de fornecer o escore total, o instrumento tem outros três indicadores relativos a três fatores: a) Fator Dieta (FD), que reflete a esquiva patológica de alimentos calóricos, excessiva preocupação com a boa forma corporal e aparência estética, relacionando-se com aspectos da imagem corporal; b) Fator Bulimia (FB), sendo relacionado com transtornos da imagem corporal, comportamento bulímico e com o peso corporal aumentado; e c) Controle Oral (CO), que reflete o autocontrole sobre a comida, assim como a consciência da pressão social para obter peso. Assim, este instrumento é capaz de predizer riscos que o indivíduo possa ter para desenvolver transtornos alimentares, em caso de padrão alimentar anormal.

2) Escala de Estresse Percebido [EPS-10] (Reis, Hino, \& Añez, 2010). Instrumento composto por 10 itens, respondidos em escala tipo Likert de cinco pontos (nunca, quase nunca, às vezes, pouco frequente, muito frequente). As perguntas são feitas a respeito de sentimentos e pensamentos durante os últimos 30 dias, como, por exemplo: Com que frequência você sentiu que foi incapaz de controlar coisas importantes na sua vida; Com que frequência você foi capaz, de controlar irritações na sua vida?.

O resultado não é uma medida critério-concorrente. No entanto, os escores podem ser comparados com a tabela normativa da população americana (Cohen, Kamarck, \& Mermelstein, 1983), sendo considerado estressado para o sexo masculino quem tem escore acima de 12,10, e o sexo feminino, acima de 13,70. Pode também ser comparado com a população de professores do Sul do Brasil (Reis et al., 2010), sendo estressados indivíduos com pontuação acima de 16,30 para o sexo masculino, e 18,30 para o sexo feminino. Ambos os critérios foram usados neste estudo. Em artigo mais 
recente, Machado, Damásio, Borsa e Silva (2014) também confirmaram as evidências de validade e precisão este instrumento.

3) Silbouette Matching Task (Stunkard, Sorenson, \& Schulsinger, 1983; adaptado para o Brasil por Marsh \& Roche, 1996). Avalia satisfação e insatisfação com a imagem corporal. É composto por 12 silhuetas em escala progressiva (de mais magro a mais gordo) e dividido em silhuetas femininas e masculinas, conforme o sexo da pessoa avaliada. O participante deve assinalar qual silhueta ele considera ser como a atual (SA) e qual seria a silhueta ideal (SI). Assinalar a mesma silhueta para SA e SI indica que há satisfação da imagem corporal; caso haja diferenças, há insatisfação da imagem corporal, sendo que, se a pontuação for positiva, significa que a pessoa deseja emagrecer e, se a pontuação for negativa, indica-se que a pessoa quer engordar.

4) Inventário de Autoestima (Coopersmith, 1967; adaptado para o português do Brasil por Okazaki, 2004). Tem 25 itens dicotômicos, em que a pessoa assinala verdadeiro ou falso conforme avaliar que a sentença se aplica à sua realidade. Como exemplos de itens, tem-se: Existem várias coisas em mim que eu mudaria se pudesse; Integro-me facilmente em qualquer ambiente; Ninguém pode contar comigo. Há um gabarito do que seria a resposta adequada para cada item. Para cada resposta correta, marca-se um ponto. Somam-se as respostas certas, multiplica-se por quatro, o que gerará um valor percentual. A classificação é dada conforme esse percentual, sendo: autoestima baixa $(<50 \%)$; autoestima normal (entre $51 \%$ e $75 \%$ ); e autoestima alta $(>76 \%$ ).

\section{Procedimento}

As escolas foram informadas sobre os objetivos do estudo, como os instrumentos seriam aplicados e em qual horário isso seria feito. Após esses esclarecimentos, foi assinado o Termo de Aceitação da Instituição. O projeto, então, foi encaminhado e aprovado pelo Comitê de Ética em Pesquisa (Parecer n. ${ }^{\circ}$ 215/2010/ CP, registro 383, emitido pela Universidade do Contestado, PR). Os pais e responsáveis foram contatados e, após compreenderem os objetivos do estudo, riscos e benefícios, assinaram o Termo de Consentimento Livre e Esclarecido. A coleta de dados foi feita de forma coletiva, em sala de aula, em horários disponibilizados pelos professores, por um aplicador treinado. Em caso de dúvidas, o aplicador poderia respondê-las, sem conduzir o participante a emitir determinada resposta.

Os dados foram computados segundo as instruções de correção de cada instrumento. Com o auxílio do software estatístico SPSS ${ }^{\circledR}$ 18.0, encontrou-se a distribuição normal (teste de Kolmogorov-Smirnov) e homogênea dos dados não dicotômicos (teste de Levene), indicando a possibilidade de análise estatística paramétrica. Foi feita a análise estatística descritiva, calculando-se a frequência, percentual, média e desvio padrão das variáveis.

Posteriormente, foram realizadas a análise de rede (Borsboom \& Cramer, 2013). Esta análise representa o padrão de associação de variáveis em um objeto gráfico bidimensional. A representação é formada por nodos e linhas, os quais representam as variáveis analisadas e as relações entre elas, respectivamente. A disposição das variáveis é definida de modo que: a) variáveis relacionadas se atraem e as não relacionadas expelem-se; b) quanto mais central é uma variável, mais relacionada às demais; c) linhas representam a associação ponderada entre os nodos, sendo positivas ou negativas. Foram analisadas as redes de correlação e correlação parcial (controlando-se o efeito multivariado), tendo nos valores de $r^{2}$ uma medida ponderada de efeito. Ainda, foram calculadas medidas de conectividade (número de ligações entre dois pares de variáveis), proximidade (número de conexões observadas em razão de todas as possíveis) e força (média dos pesos das associações com demais variáveis no sistema) entre as variáveis.

\section{Resultados}

Inicialmente, são apresentados os resultados descritivos de cada variável, para caracterizar a amostra nos instrumentos, como apresentado na Tabela 1.

É possível perceber que todos os participantes estavam com estresse acima da média da população norte-americana e sul-brasileira. A maioria das meninas estava dentro do peso normal, com IMC na faixa normal; mas houve, mais meninas abaixo do peso, comparando com o percentual de $17,8 \%$ dos meninos (Tabela 1).

Houve diferenças de gênero em relação à imagem corporal, com a maioria das meninas desejando emagrecer e a maioria dos meninos querendo engordar. Identificou-se um alto nível de insatisfação corporal na amostra, especialmente nos meninos. Constatou-se mais disfunção dos padrões alimentares nas meninas do que nos meninos. Estes apresentaram uma autoestima 
Tabela 1

Estresse, indice de massa corporal, padrão alimentar, imagem corporal e autoestima em adolescentes e jovens de 14 a 20 Anos $(N=238)$

\begin{tabular}{|c|c|c|c|c|}
\hline Sexo & Feminino & Masculino & Feminino & Masculino \\
\hline Variáveis & $\mathrm{n}(\%)$ & $\mathrm{n}(\%)$ & $\begin{array}{c}\% \\
\text { acumulada }\end{array}$ & $\begin{array}{c}\% \\
\text { acumulada }\end{array}$ \\
\hline \multicolumn{5}{|l|}{ Estresse } \\
\hline Abaixo da média & $0(0,0)$ & $0(0,0)$ & 0 & 0 \\
\hline Acima da média & $148(100,0)$ & $90(100,0)$ & 100 & 100 \\
\hline \multicolumn{5}{|l|}{ Índice de Massa Corporal } \\
\hline Abaixo & $40(27,0)$ & $16(17,8)$ & 27 & 17,8 \\
\hline Normal & $98(66,2)$ & $68(75,6)$ & 93,2 & 93,3 \\
\hline Sobrepeso & $9(6,1)$ & $6(6,7)$ & 99,3 & 100 \\
\hline Obesidade & $1(0,7)$ & $0(0,0)$ & 100 & 100 \\
\hline \multicolumn{5}{|l|}{ Padrão alimentar } \\
\hline Normal & $114(77,0)$ & $78(89,8)$ & 77 & 86,8 \\
\hline Anormal & $34(23,0)$ & $12(13,2)$ & 100 & 100 \\
\hline \multicolumn{5}{|l|}{ Imagem Corporal } \\
\hline Satisfeito & $35(23,6)$ & $17(18,9)$ & 23,6 & 18,9 \\
\hline Insatisfeito querendo emagrecer & $92(62,2)$ & $29(32,2)$ & 85,8 & 51,1 \\
\hline Insatisfeito querendo engordar & $21(14,2)$ & $44(48,9)$ & 100 & 100 \\
\hline \multicolumn{5}{|l|}{ Autoestima } \\
\hline Baixa & $31(20,9)$ & $13(14,3)$ & 20,9 & 14,3 \\
\hline Média & $99(66,9)$ & $66(72,5)$ & 87,8 & 86,8 \\
\hline Alta & $18(12,2)$ & $12(13,2)$ & 100 & 100 \\
\hline
\end{tabular}

dentro da média, assim como as meninas; mas, entre aqueles com baixa autoestima, houve prevalência de meninas (Tabela 1).

Foi feita a análise de rede para verificar, de forma sistêmica, as relações entre as variáveis. Na Figura 1 (grafo 1), são apresentadas as relações bivariadas padronizadas, de modo a resumir uma grande quantidade de informação. Posteriormente, foi feita a rede de correlações parciais (Figura 1, grafo 2). Este método visa a identificar relações mais estáveis no sistema analisado, por meio do controle multivariado sobre cada relação par a par. Dessa forma, a rede possui propriedades markovianas, em que cada linha representa a relação exclusiva entre cada par no sistema.

Nesse sistema, a variável dieta apresenta maior relação com as demais do sistema. Notam-se as relações entre as variáveis: Fator Bulimia e Fator Dieta $(r=$
0,53), Imagem Corporal e IMC $(r=0,48)$, Autoestima e Estresse Percebido $(r=-0,50)$. A variável idade ficou isolada, não estando relacionada significativamente com nenhuma outra (Figura 1).

As relações mais salientes no sistema foram observadas entre as variáveis: imagem corporal e IMC ( $(\tilde{\mathrm{n}}=$ $0,52)$, entre fator dieta e fator bulimia $(\tilde{n}=0,51)$ e entre autoestima e estresse percebido $(\tilde{\mathrm{n}}=-0,48)$. Percebe-se que foram as mesmas relações observadas no grafo 1 da Figura 1, porém, com valores diferentes de correlação, já que, nesse caso, utilizou-se a correlação parcial. Em ambas as redes de correlações, a imagem corporal e o sexo apresentaram correlações negativas, indicando que as meninas têm maior preocupação com a imagem corporal. Nesse sistema, as variáveis IMC e imagem corporal emergem como mais relevantes no sistema. $\mathrm{E}$, novamente, a idade ficou isolada, não apresentando 

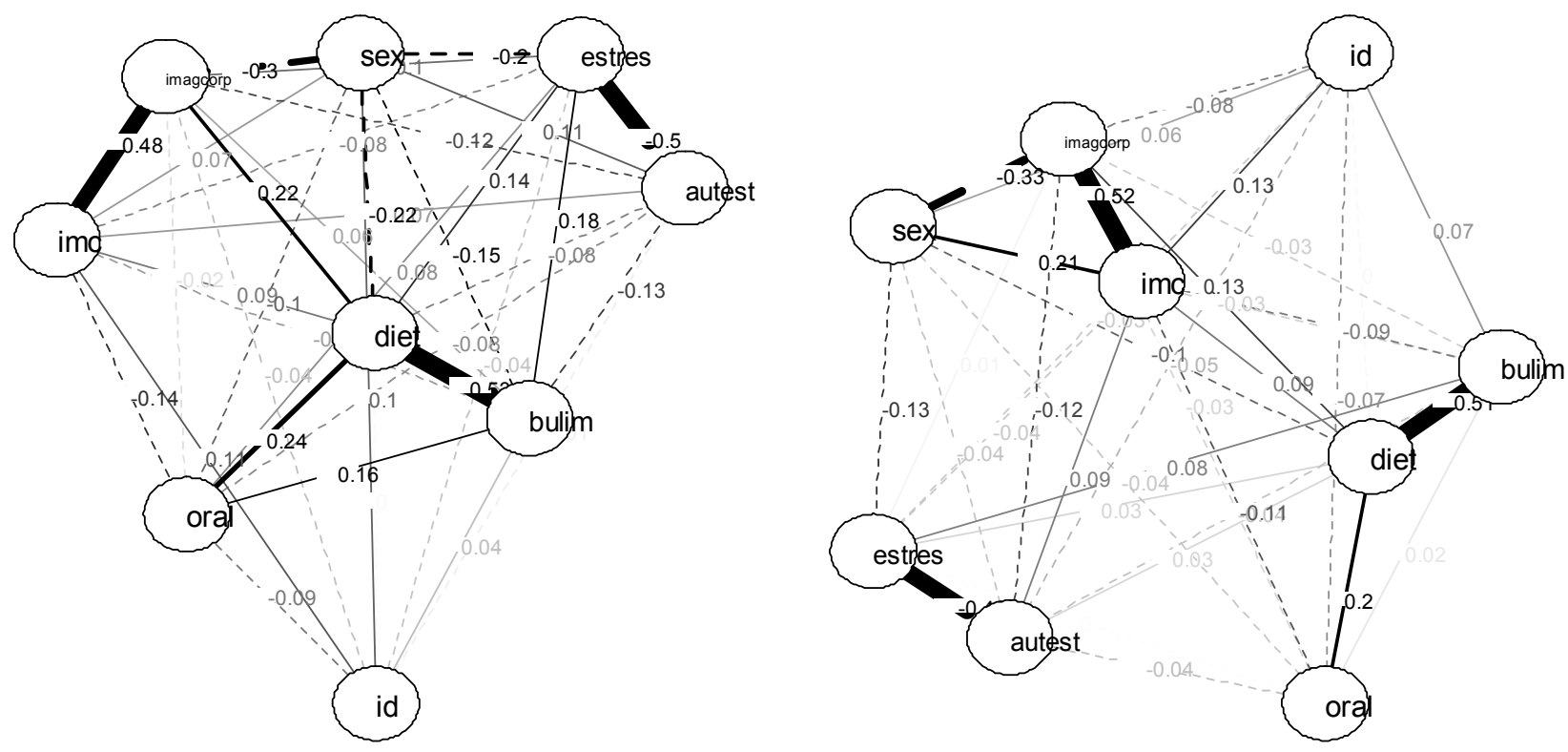

Legenda: Autoest - autoestima; estres - estresse; sex - sexo; imagcorp - imagem corporal; imc - índice de massa corporal; bulim - fator bulimia; diet - fator dieta; oral - fator controle oral; id - idade. Linha mais grossa indica associação mais forte.

Nota. Os valores expressam o valor padronizado da associação entre variáveis. Linhas com valor absoluto igual ou maior de 0,15 foram destacadas para melhor visualização.

Figura 1. Rede de correlações bivariadas (grafo 1) e rede de correlações parciais (grafo 2) de indicadores de estresse, Índice de Massa Corporal, padrão alimentar, imagem corporal e autoestima de adolescentes e jovens $(N=238)$.

relações significativas com nenhuma das variáveis (Figura 1).

$\mathrm{Na}$ Figura 2, são apresentadas as medidas de conectividade, proximidade e força para a rede de correlações parciais de indicadores de estresse, índice de massa corporal, padrão alimentar, imagem corporal e autoestima na amostra avaliada.

É possível observar que o IMC foi a variável com maior betweeness (conectividade), ou seja, é a variável que mais faz ligações com outras. A imagem corporal apresentou mais closeness (proximidade), possuindo menor distância das outras variáveis porque representa o número de conexões observadas em razão de todas as possíveis. O IMC e a imagem corporal tiveram mais strength (força), já que possuíram as correlações mais fortes com as demais variáveis do sistema (Figura 2).

\section{Discussão}

A preocupação com o peso, a insatisfação corporal, a busca pelo corpo ideal e a realização de dietas são indicadores para o comportamento alimentar inadequado (Cordás et al., 2007; Fortes et al., 2013; Lima et al., 2012). Essas relações foram observadas nesta amostra, em que 19,3\% estavam em risco para transtornos alimentares, segundo o EAT-26. A partir do presente estudo, pode-se indicar que, descontentes com a imagem corporal, as meninas podem apresentar comportamentos alimentares de risco, relacionados a uma dieta restritiva e à bulimia. Assim, deve-se atentar para essas variáveis, que podem ser inseridas em uma avaliação psicológica de rastreio, com instrumentos como os aqui aplicados, e acompanhamento psicológico, por exemplo. A identificação de uma insatisfação corporal nessa faixa etária pode ser indicativa de risco para as relações com possíveis transtornos alimentares e dismórficos, como apresentado neste estudo.

Confirmando esse risco, o fator bulimia do EAT-26 apresentou a maior média nesta amostra. A principal característica da bulimia é a ingestão alimentar incontrolável, seguido por algum tipo de purgação. A literatura aponta que a pessoa com bulimia sente medo de engordar; portanto, costuma restringir sua ingestão de alimentos. Entretanto, mesmo seguindo uma dieta 

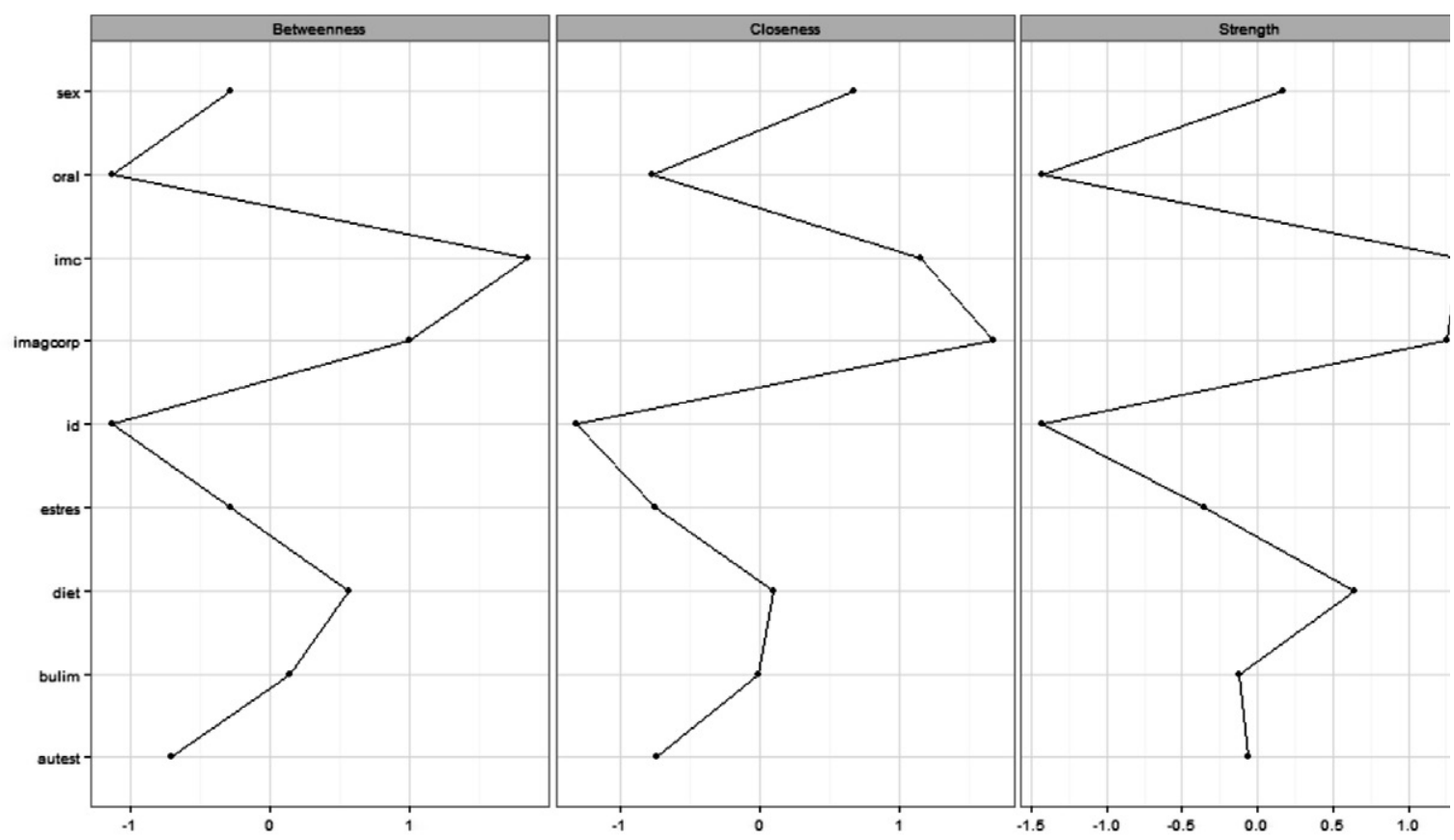

Legenda: Autoest - autoestima; estres - estresse; sex - sexo; imagcorp - imagem corporal; imc - índice de massa corporal; bulim - fator bulimia; diet - fator dieta; oral - fator controle oral; id - idade.

Figura 2. Medidas de conectividade, proximidade e força para a rede de correlações parciais de indicadores de estresse, Índice de Massa Corporal, padrão alimentar, imagem corporal e autoestima em uma amostra de adolescentes e jovens $(N=238)$.

restritiva, perde o controle e apresenta atos de compulsão alimentar (Herpertz-Dahlmann, 2008; Urgesi et al., 2011).

Outros estudos afirmam que jovens com bulimia apresentam excesso de peso (Alvarenga et al., 2011; Gomes et al., 2010; Rossi, Moreira, \& Rauen, 2008). Importante notar nesse contexto que meninas são mais propensas a apresentar transtornos alimentares (Alvarenga et al., 2011; Lima et al., 2012; Scherer et al., 2010), resultado também observado nesta pesquisa, sendo que o comportamento alimentar inadequado foi identificado em 34 meninas (23\%), contra 12 meninos $(13,2 \%)$. Além disso, essa relação também foi observada na análise de rede, em que o sexo foi fortemente relacionado ao fator dieta e indiretamente relacionado aos fatores bulimia e controle oral.

Contudo, como 93,2\% das meninas e 93,3\% dos meninos do presente estudo estavam na faixa de peso abaixo e dentro da normalidade, não foi encontrada a relação entre maior peso e maior tendência à bulimia, identificada em algumas pesquisas, em que meninas obesas apresentaram maior índice de comportamentos purgativos, quando comparadas às meninas com baixo peso (Fortes et al., 2013). Assim, outras variáveis podem estar afetando essa relação. E essa falta de associação entre peso e bulimia é uma limitação no diagnóstico da bulimia, pois, em alguns casos, não existe uma perda significativa de peso e, por consequência, redução no IMC (Fernandes, 2006; Keski-Rahkonen et al., 2008).

Outra variável importante nessas relações é o estresse (Gonçalves et al., 2013), que foi identificado em toda a amostra do presente estudo, em níveis acima da média. Essa condição de altos níveis de estresse, como já mencionado, aumenta a liberação do cortisol, estimulando a ingestão de alimentos e, por consequência, a compulsão alimentar (Gluck et al., 2001; Gonçalves et al., 2013). Essa situação se agrava no caso das meninas, que possuem maiores oscilações hormonais e estão sujeitas a condições culturais, como o acúmulo de tarefas cotidianas (Gonzaga, Macedo, \& Lipp, 2014; Marques, Gasparotto, \& Coelho, 2015; Schermann et al., 2014). Essa relação de gênero foi confirmada nesta amostra, em que as meninas apresentaram maiores níveis de estresse.

De outro lado, observou-se que os altos níveis de estresse apresentaram uma correlação inversamente 
proporcional com a autoestima no presente estudo, de modo que esses adolescentes sob estresse tendem a apresentar menor autoestima. Essa relação tem sido apontada por outros estudos (Moksnes, Bradley Eilertsen, \& Lazarewicz, 2016; Murray et al., 2013), tornando a questão da prevenção do estresse de relevância para romper um possível ciclo entre estresse, baixa autoestima, insatisfação com o corpo e desenvolvimento de psicopatologias. Contudo, a autoestima por si só não se correlacionou ao comportamento alimentar compulsivo, assim como não foram encontradas relações entre a autoestima e outro tipo de compulsão, no caso, por compras (Medeiros, Diniz, Costa, \& Pereira, 2015).

Por sua vez, os resultados sugerem relações mais próximas entre a imagem corporal e o IMC, sendo central na problemática da saúde dos adolescentes. No presente estudo, os meninos apresentaram IMC maior que as meninas, o que é esperado, tendo em vista a maior massa magra e maior altura masculina (Powers \& Howley, 2000). Entretanto, esses resultados precisam ser analisados com cautela. Yang et al. (2012) publicaram um estudo realizado com mais de 100.000 pessoas, mostrando que existem diferenças genéticas, com genótipos específicos que são associados com fenótipos que causam variabilidade do IMC. Isso indica que há influências genéticas no IMC, e que, portanto, podem variar, sendo homens ou mulheres.

Ainda em relação à imagem corporal, de acordo com as análises aqui apresentadas, meninas indicaram desejo de perder peso, e os garotos, em sua maioria, gostariam de aumentar sua massa muscular, confirmando estudos da área (Biolcati et al., 2016; Gondoli et al., 2012). Pritchard e Cramblitt (2014) focalizam ainda importante aspecto não aferido por esta pesquisa, mas que pode contribuir para compreensão do resultado no sexo masculino: homens que assistem mais televisão e leem revistas de saúde masculinas apresentam maior tendência querer um corpo musculoso. Assim, vê-se que, durante a adolescência e o início da vida adulta, há uma preocupação com o corpo, porém, em caso de não aceitação do corpo, pode acarretar nos jovens altos índices de insatisfação corporal, como os que foram constatados neste estudo pelo Silhouette Matching Task, com $76,4 \%$ das meninas e $81,1 \%$ dos meninos insatisfeitos com o corpo.

\section{Considerações Finais}

Este estudo obteve indicadores de insatisfação com a imagem corporal e comportamentos alimentares inadequados em uma amostra grande de adolescentes e jovens adultos, apontando para a necessidade de ações preventivas para essa população. A prevenção gera diminuição de custos em saúde pública e minimiza os efeitos nocivos de um possível transtorno psiquiátrico (Fries, Koop, Sokolov, Beadle, \& Wright, 1998; Sánchez-Carracedo, Neumark-Sztainer, \& López-Guimerà, 2012).

Apesar do tamanho da grande amostra, este estudo foi realizado com estudantes de escolas particulares, afetando a generalização dos resultados; portanto, sugere-se que novas pesquisas sejam feitas, comparando-se escolas públicas e privadas, além de analisar as possíveis diferenças entre as faixas etárias incluídas na adolescência e os jovens adultos. Há também uma limitação dos instrumentos e variáveis analisadas, sendo que pesquisas futuras podem estudar outras variáveis relevantes, como o temperamento (Anzman \& Birch, 2009) e o coping (enfrentamento) (Park \& Iacocca, 2014), por exemplo, com enfoque motivacional, para melhor compreender essas relações (Verstuyf, Patrick, Vansteenkiste, \& Teixeira, 2012).

A despeito dessas limitações, pode-se considerar que o estudo trouxe avanços em termos metodológicos, por incluir muitas variáveis em uma única análise, além da aplicação da análise de rede para o contexto clínico, saindo, assim, de uma perspectiva dualista, para uma visão integrativa. Esse tipo de análise é recente e vem se desenvolvendo ao redor do mundo e em diferentes áreas de conhecimento. Quando aplicada nos estudos de fenômenos psicológicos, a análise de rede mostra-se rica por verificar a relação sistêmica entre as variáveis, em que, ao se alterar uma variável, as demais por consequência também poderão ser mudadas.

Pode-se considerar que os adolescentes e jovens esta amostra apresentaram condições de risco para transtornos alimentares descritas pela literatura: insatisfação com imagem corporal, tendências a transtornos alimentares, com particularidades nas diferenças entre os sexos. Essas diferenças devem orientar as propostas de intervenção, considerando as influências midiáticas e culturais que essa população sofre em termos de ideais de beleza física.

Como forma de evitar a possível ocorrência dos transtornos alimentares, como anorexia e bulimia, sugere-se um trabalho preventivo, de avaliação e encaminhamentos para intervenção. Justamente por ser uma condição multideterminada, essas ações devem ser multidisciplinares, com a atuação de profissionais da Psiquiatria, Psicologia, Nutrição, Educação Física, 
para citar as principais. Para as meninas, sugere-se a avaliação e intervenções sobre as variáveis que se mostraram com maior força de associação, tais como preocupação em emagrecer, estresse e autoestima. Orientações sobre alimentação e nutrição, programas de controle de estresse e atividades que visem aos cuidados com autoestima são sugeridos. Quanto aos meninos, a atenção deve estar voltada à necessidade de aumentar a massa corporal, com comportamentos que tendam à vigorexia. Devem ser feitas orientações sobre práticas adequadas de exercício físico e estímulo à alimentação e nutrição adequada. Espera-se que intervir nas variáveis mais centrais indicadas pelas análises impacte nas variáveis de desfecho (IMC, comportamento alimentar), evitando a evolução para os transtornos alimentares.

\section{Referências}

Alvarenga, M. D. S., Scagliusi, F. B., \& Philipp, S. T. (2011). Comportamento de risco para transtorno alimentar em universitárias brasileiras. Revista de Psiquiatria Clinica, 38(1), 3-7. doi: 10.1590/ S0101-60832011000100002

Anzman, S. L., \& Birch, L. L. (2009). Low inhibitory control and restrictive feeding practices predict weight outcomes. The Journal of Pediatrics, 155(5), 651-656. doi: 10.1016/j.jpeds.2009.04.052

American Pscyhological Association (2014). Manual Diagnóstico e Estatístico de Transtornos Mentais - DSM5 (M. I. C. Nascimento, Trad.). Porto Alegre: Artmed.

Bighetti, F., Santos, C. B., Santos, J. E., \& Ribeiro, R. P. P. (2004). Tradução e avaliação do Eating Attitudes Test em adolescentes do sexo feminino de Ribeirão Preto. Jornal Brasileiro de Psiquiatria, 53(6), 339-346. doi: 10.11606/D.22.2003.tde-12042004-234230

Biolcati, R., Ghigi, R., Mameli, C., \& Passini, S. (2016). What can I do with my body? Boys and girls facing body dissatisfaction. International Journal of Adolescence and Youth, 3843(May), 1-13. doi: 10.1080/02673843.2016.1167748

Borsboom, D., \& Cramer, A. O. J. (2013). Network Analysis: An integrative approach to the structure of Psychopathology. Annual Review of Clinical Psychology, 9(1), 91-121. doi: 10.1146/ annurev-clinpsy-050212-185608
Claudat, K., White, E. K., \& Warren, C. S. (2016). Acculturative stress, self-esteem, and eating pathology in Latina and Asian American female college students. Journal of Clinical Psychology, 72(1), 88-100. doi: 10.1002/jclp.22234

Cohen, S., Kamarck, T., \& Mermelstein, R. (1983). A global measure of perceived stress. Journal of Health and Social Behavior, 24(4), 385-396. Recuperado de http://www.psy.cmu.edu/ scohen/ globalmeas83.pdf

Conti, M. A., Bertolin, M. N. T., \& Peres, S. V. (2010). A mídia e o corpo: $\mathrm{O}$ que o jovem tem a dizer? Ciência \& Saúde Coletiva, 15(4), 2095-2103. doi: 10.1590/S1413-81232010000400023

Coopersmith, S. (1967). The antecedents of self-esteem (Vol. 1). San Francisco: W. H. Freeman \& Co.

Cordás, T., Oliveira, G., Figueired, M., \& Didone, N. (2007). Transtornos alimentares: Epidemiologia, etiologia e classificação. Revista Nutrição Profissional, 11(1), 12-20. Recuperado de http://www.bib. unesc.net/arquivos/70000/73600/11_73615.htm

Costarelli, V., \& Patsai, A. (2012). Academic examination stress increases disordered eating symptomatology in female university students. Eating and Weight Disorders-Studies on Anorexia, Bulimia and Obesity, 17(3),164-169.

De Vries, D. A., Peter, J., De Graaf, H., \& Nikken, P. (2016). Adolescents' social network site use, peer appearance-related feedback, and body dissatisfaction: Testing a mediation model. Journal of Youth and Adolescence, 45(1), 211-224. doi: 10.1007/ s10964-015-0266-4

Dumith, S. C., \& Farias Júnior, J. C. (2010). Sobrepeso e obesidade em crianças e adolescentes: Comparação de três critérios de classificação baseados no índice de massa corporal. Revista Panamericana de Salud Pública, 28(1), 30-35. doi: 10.1590/ S1020-49892010000700005

Fernandes, M. H. (2006). Transtornos alimentares: Anorexia e bulimia. São Paulo: Casa do Psicólogo.

Fortes, L., Amaral, A., Conti, M., Cordas, T., \& Ferreira, M. [s.d.]. Qualidades psicométricas do Eating Attitudes Test (EAT-26) para a população adolescente masculina brasileira. Psicologia: Reflexão e Crítica. (no prelo). 
Fortes, L. S., Almeida, S. S., \& Ferreira, M. E. C. (2012). Impacto de variáveis antropométricas sobre a insatisfação corporal e o comportamento alimentar em jovens atletas. Jornal Brasileiro de Psiquiatria, 61(4), 235-241. doi: 10.1590/S0047-20852012000400007

Fortes, L. S., Amaral, A. C. S., Almeida, S. S., \& Ferreira, M. E. C. (2013). Efeitos de diversos fatores sobre o comportamento alimentar de adolescentes. Ciência \& Saúde Coletiva, 18(11), 3301-3310. doi: 10.1590/ S1413-81232013001100020

Fortes, L. S., Amaral, A. C. S., \& Ferreira, M. E. C. (2013). Comportamento alimentar inadequado em adolescentes de Juiz de Fora. Temas em Psicologia, 21(2), 403-410. doi: 10.9788/TP2013.2-08

Fortes, L. S., Ferreira, M. E. C., Costa, P. R. F., Lira, H. A. A. S., Andrade, J., \& Silva, A. L. A. G. (2015). Comparação do comportamento de risco para transtornos alimentares entre adolescentes atletas e não atletas. Jornal Brasileiro de Psiquiatria, 64(4):296-302.

Fries, J. F., Koop, C. E., Sokolov, J., Beadle, C. E., \& Wright, D. (1998). Beyond health promotion: Reducing need and demand for medical care. Health Affairs, 17(2), 70-84. doi: 10.1377/hlthaff.17.2.70

Garner, D. M., Olmsted, M. P., Bohr, Y., \& Garfinkel, P. E. (1982). The Eating Attitudes Test: Psychometric features and clinical correlates. Psychological Medicine, 12(1), 871-878. Recuperado de http://www.eat-26. com/Docs/Garner-EAT-26-1982.pdf

Gluck, M. E., Geliebter, \& Satov, T. (2001). Night eating syndrome is associated with depression, low self-esteem, reduced daytime hunger, and less weight loss in obese outpatients. Obesity Research, 9(4), 264-267. doi: 10.1038/oby.2001.31

Gomes, J. P., Legnani, E., Legnani, R. F. S., Gregório, N. P., \& Souza, R. K. (2010). Associação entre comportamento alimentar, consumo de cigarro, drogas e episódios depressivos em adolescentes. Revista de Nutrição, 23(5), 755-762. doi: 10.1590/ S1415-52732010000500006

Gonçalves, J. D. A., Moreira, E. A. M., M, E. B. S., \& Fiates, G. M. R. (2013). Transtornos alimentares na infância e na adolescência. Revista Paulista de Pediatria, 31(1), 96-103. doi: 10.1590/ S0103-05822013000100016
Gondoli, D. M., Corning, A. F., Salafia, E. H. B., Bucchianeri, M. M., \& Fitzsimmons, E. E. (2012). Heterosocial involvement, peer pressure for thinness, and body dissatisfaction among young adolescent girls. Body Image, 8(2), 143-148. doi: h10.1016/j.bodyim.2010.12.005.Heterosocial

Gonzaga, L. R. V., Macedo, A. G. de, \& Lipp, M. E. N. (2014). Avaliação das variáveis escolha profissional e vocação no nível de stress de alunos do Ensino Médio. Em C. Vichi, E. Huriwara, H. Sadi, \& L. Postalli (Eds.), Comportamento em Foco 3 (pp. 189-202). São Paulo: Associação Brasileira de Psicologia e Medicina Comportamental.

Grilo, C. M. (2014). Eating and Weight Disorders. New York: Psychology Press.

Gustafsson, S. A., Edlund, B., Davén, J., Kjellin, L., \& Norring, C. (2010). Perceived expectations in daily life among adolescent girls suffering from an eating disorder: A phenomenographic study. Eating Disorders, 18(1),25-42. doi:10.1080/10640260903439524

Herpertz-Dahlmann, B. (2008). Adolescent eating disorders: Update on definitions, symptomatology, epidemiology, and comorbidity. Child and Adolescent Psychiatric Clinics of North America, 18(1), 31-47. doi: 10.1016/j.chc.2008.07.005

Jackson, T., \& Chen, H. (2010). Sociocultural experiences of bulimic and non-bulimic adolescents in a school-based Chinese sample. Journal of $A b$ normal Child Psychology, 38(1), 69-76. doi: 10.1007/ s10802-009-9350-0

Keski-Rahkonen, A., Hoek, H. W., Linna, M. S., Raevuori, A., Sihvola, E., Bulik, C. M., ... Kaprio, J. (2008). Incidence and outcomes of bulimia nervosa: A nationwide population-based study. Psychological medicine, 39(5), 823-31. doi: 10.1017/ S0033291708003942

Leite, N., Milano, G. E., Lopes, W. A., Tanaka, J., Dressler, V. F., \& Radominski, R. B. (2008). Comparação entre critérios para índice de massa corporal na avaliação nutricional em escolares. Revista da Educação Física/UEM, 19(4), 557-563. doi: 10.4025/ reveducfis.v19i4.4709

Lima, N. L., Rosa, C. D. O. B., \& Rosa, J. F. V. (2012). Identificação de fatores de predisposição aos transtornos alimentares: Anorexia e bulimia em adolescentes de Belo Horizonte, Minas Gerais. 
Estudos e Pesquisas em Psicologia, 12(2), 360-378. doi: 10.12957/epp.2012.8267

Machado, W. L., Damásio, B. F., Borsa, J. C., \& Silva, J. P. da. (2014). Dimensionalidade da escala de estresse percebido (Perceived Stress Scale, PSS-10) em uma amostra de professores. Psicologia: Reflexão e Crítica, 27(1), 38-43. doi: /10.1590/ S0102-79722014000100005

Machado, W. L., Vissoci, J., \& Epskamp, S. (2015). Análise de rede aplicada à Psicometria e à avaliação psicológica. Em C. S. Hutz, D. R. Bandeira \& C. M. Trentini (Eds.), Psicometria (pp. 125-146). Porto Alegre.

Marques, C. P., Gasparotto, S., \& Coelho, R. W. (2015). Fatores relacionados ao nível de estresse em adolescentes estudantes: Uma revisão sistemática. Salusvita, 34(1), 99-108. Recuperado de http:// www.usc.br/biblioteca/salusvita/salusvita_v34_ n1_2015_art_07.pdf

Marsh, H. W., \& Roche, L. A. (1996). Predicting self-steem from perceptions of actual and ideal ratings of body fatness: Is there only one ideal ratings of body fatness: Is there only one ideal supermodel. Research Quartly for Exercise and Sport, 67(1), 3-23. doi: 10.1080/02701367.1996.10607921

Medeiros, F. G., Diniz, I. S. F. N., Costa, F. J., \& Pereira, R. C. F. (2015). Influência de estresse, materialismo e autoestima na compra compulsiva de adolescentes. Revista de Administração Contemporânea, 19(2), 137-156. doi: 10.1590/1982-7849rac20151553

Miranda, V. P. N., Conti, M. A., Bastos, R., \& Ferreira, M. E. C. (2011). Insatisfação corporal em adolescentes brasileiros de municípios de pequeno porte de Minas Gerais. Jornal Brasileiro de Psiquiatria, 60(3), 190-197. doi: 10.1590/S0047-20852011000300007

Moksnes, U. K., Eilertsen, M. E. B., \& Lazarewicz, M. (2016). The association between stress, self-esteem and depressive symptoms in adolescents. Scandinavian Journal of Psychology, 57(1), 22-29. doi: 10.1111/ sjop.12269

Murray, K., Rieger, E., \& Byrne, D. (2013). A longitudinal investigation of the mediating role of self-esteem and body importance in the relationship between stress and body dissatisfaction in adolescent females and males. Body Image, 10(4), 544-551. doi: 10.1016/j.bodyim.2013.07.011
National Institutes of Health. (2000). The practical guide. identification, evaluation, and treatment of overweight and obesity in adults, 26-7. Recuperado de http://www. nhlbi.nih.gov/files/docs/guidelines/prctgd_c.pdf

Nunes, M. A., Bagatini, L. F., Abuchaim, A. L., Kunz, A., Ramos, D., Silva, J. A., ... Pinheiro, A. (1994). Distúrbios da conduta alimentar: Consideraçöes sobre o Teste de Atitudes Alimentares (EAT). Revista ABPAPAL, 16(1), 7-10.

Okazaki, F. (2004). Relação entre auto-estima, o nivel técnico, o tempo de prática e o resultado da competição de atletas de voleibol feminino da categoria mirim. Faculdades Dom Bosco, Curitiba-Paraná.

Park, C. L., \& Iacocca, M. O. (2014). A stress and coping perspective on health behaviors: Theoretical and methodological considerations. Anxiety, Stress, \& Coping, 27(2), 123-137. doi: 10.1080/10615806.2013.860969

Powers, S. K., \& Howley, E. T. (2000). Teoria e aplicação ao condicionamento e ao desempenho ( $3^{\mathrm{a}}$ ed., M. Ikeda, Trad.). Barueri: Manole.

Pritchard, \& Cramblitt. (2014). Media influence on drive for thinness and drive for musculaturity. Sex Roles, 71(5), 2018-218. doi: 10.1007/s11199-014-0397-1

Rawal, A., Williams, J. M. G., \& Park, R. J. (2011). Effects of analytical and experiential self-focus on stress-induced cognitive reactivity in eating disorder psychopathology. Behaviour Research and Therapy, 49(10), 635-645. doi: 10.1016/j.brat.2011.06.011

Reis, R. S., Hino, A. A. F., \& Añez, C. R. R. (2010). Perceived stress scale: Reliability and validity study in Brazil. Journal of Health Psychology, 15(1), 107-114. doi: $10.1177 / 1359105309346343$

Rossi, A., Moreira, E. A. M., \& Rauen, M. S. (2008). Determinantes do comportamento alimentar: Uma revisão com enfoque na família. Revista de Nutricao, 21(6), 739-748. doi: 10.1590/ S1415-52732008000600012

San Martini, M. C., Assumpção, D., Barros, M. B. A., Canesqui, A. M., \& Barros Filho, A. A. (2016). Are normal-weight adolescents satisfied with their weight? São Paulo Medical Journal, 134(3), 219-227. doi: 10.1590/1516-3180.2015.01850912

Sánchez-Carracedo, D., Neumark-Sztainer, D., \& López-Guimerà, G. (2012). Integrated prevention of obesity and eating disorders: Barriers, developments 
and opportunities. Public Health Nutrition, 15(12), 2295-2309. doi: 10.1017/S1368980012000705

Santos, N. D. O., Marques, V. G., Maihara, A., Rosana, G., Benute, G., Cristina, M., \& Lucia, S. (2012). Vigorexia, uso de anabolizantes e a (não) procura por tratamento psicológico: Relato de experiência. Psicologia Hospitalar, 10(1), 2-15. Recuperado de http:/ / pepsic.bvsalud.org/scielo.php?script=sci_arttext\& pid $=$ S1677-74092012000100002

Scherer, F. C., Martins, C. R., Pelegrini, A., Matheus, S. C., \& Petroski, E. L. (2010). Imagem corporal em adolescentes: Associação com a maturação sexual e sintomas de transtornos alimentares. Jornal Brasileiro de Psiquiatria, 59(3), 198-202. doi: 10.1590/ S0047-20852010000300005

Schermann, L. B., Béria, J. U., Jacob, M. H. V. M., Arossi, G., Benchaya, M. C., Bisch, N. K., \& Rieth, S. (2014). Estresse em adolescentes: Estudo com escolares de uma cidade do sul do Brasil. Aletheia, 160-173. Recuperado de http://pepsic. bvsalud.org/scielo.php?script=sci_abstract\&pid $=$ S1413-03942014000100012

Stice, E., \& Shaw, H. E. (2002). Role of body dissatisfaction in the onset and maintenance of eating pathology. Journal of Psychosomatic Research, 53(5), 985-993. doi: 10.1016/S0022-3999(02)00488-9

Stunkard, A. J., Sorenson, T., \& Schulsinger, F. (1983). Use of the danish adaption registry for the study of obesity and thinness. Association for Research in Nervous and Mental Disease, 60(1), 115-120.

Tiggemann, M., \& Slater, A. (2015). The role of self-objectification in the mental health of early adolescent girls: Predictors and consequences. Journal of Pediatric Psychology, 40(7), 704-711. doi: 10.1093/jpepsy/jsv021

Urgesi, C., Fornasari, L., De Faccio, S., Perini, L., Mattiussi, E., Ciano, R., ... Brambilla, P. (2011). Body schema and self-representation in patients with bulimia nervosa. International Journal of Eating Disorders, 44(3), 238-248. doi: 10.1002/eat.20816

Verstuyf, J., Patrick, H., Vansteenkiste, M., \& Teixeira, P. J. (2012). Motivational dynamics of eating regulation: A self-determination theory perspective. International Journal of Behavioral Nutrition and Physical Activity, 9(21), 1-16. doi: 10.1186/1479-5868-9-21

Yang, J., Loos, R. J. F., Powell, J. E., Medland, S. E., Speliotes, E. K., Chasman, D. I., ... Visscher, P. M. (2012). FTO genotype is associated with phenotypic variability of body mass index. Nature, 490(7419), 267-272. doi: 10.1038/nature11401

World Health Organization - WHO. (2007). The challenge of obesity in the WHO European Region and the strategies for response. Denmark: WHO Regional Office for Europe.

Recebido em: 05/09/2016

Reformulado em: 30/07/2017

Aprovado em: 10/10/2017 
Sobre os autores:

Andressa Melina Becker da Silva é doutora em Psicologia pela Pontifícia Universidade Católica de Campinas (PUC-Campinas), com estágio pós-doutoral na mesma instituição, mestre em Educação Física pela Universidade Federal do Paraná (UFPR) e professora nos cursos de graduação em Psicologia e Educação Física da Universidade de Sorocaba (UNISO).

E-mail: andressa_becker@hotmail.com

ORCID: 0000-0001-5630-7843

Wagner de Lara Machado é doutor em Psicologia pela Universidade Federal do Rio Grande do Sul (UFRGS) com estágio pós-doutoral na mesma instituição, docente do Programa de Pós-Graduação em Psicologia da Pontifícia Universidade Católica do Rio Grande do Sul (PUCRS).

E-mail:wagner.machado@pucrs.br

ORCID: 0000-0001-5555-5116

Anita Colletes Bellodi é doutora em Psicologia pela Pontifícia Universidade Católica de Campinas (PUC-Campinas); Mestre em Psicologia Experimental: Análise do Comportamento da PUC-SP; Professora do curso de graduação em Psicologia e na Residência Multidisciplinar em Saúde, Urgência e Trauma da PUC-Campinas.

E-mail: anitabellodi@gmail.com

ORCID: 0000-0002-3287-2001

Kainara Silva da Cunha é graduada em Psicologia pela Pontifícia Universidade Católica de Campinas (PUC-Campinas), mestre em Ciências da Saúde pela PUC-Campinas; Psicóloga assistencial da Clínica Escola de Psicologia da PUC-Campinas e Psicóloga Técnica do Serviço de Psicologia Aplicada da Unisal/Campinas.

E-mail: kainaracunha@gmail.com

ORCID: 0000-0002-2355-145X

Sônia Regina Fiorim Enumo é doutora em Psicologia Experimental pela Universidade de São Paulo (USP), docente orientadora do Programa de Pós-Graduação em Psicologia e do Programa de Pós-Graduação em Ciências da Saúde da Pontifícia Universidade Católica de Campinas (PUC-Campinas) e Professora Emérita na Universidade Federal do Espírito Santo (UFES).

E-mail: sonia.enumo@puc-campinas.edu.br

ORCID: 0000-0001-9038-6151

Contato com os autores:

Andressa Melina Becker da Silva

Av. das Pitangueiras, 163, Cond. Margaridas, Bloco E, ap. 128, Vossoroca

Votorantim-SP, Brasil

CEP: 18119-372 